\title{
Adaptação Brasileira e Estrutura Fatorial da Escala 240-item VIA Inventory of Strengths
}

\author{
Bruna Larissa Seibel - Universidade Federal do Rio Grande do Sul, Porto Alegre, Brasil \\ Diogo DeSousa - Universidade Federal do Rio Grande do Sul, Porto Alegre, Brasil \\ Silvia Helena Koller - Universidade Federal do Rio Grande do Sul, Porto Alegre, Brasil
}

\begin{abstract}
Resumo
Este estudo objetivou: (1) realizar a adaptação transcultural do 240-item VIA Inventory of Strengths (VIA-IS) para o Brasil; (2) investigar a estrutura fatorial do VIA-IS em uma amostra brasileira; e (3) reportar dados descritivos de escores de forças no VIA-IS nessa amostra. A adaptação da escala para uso no Brasil seguiu diretrizes padronizadas para adaptação transcultural de instrumentos psicológicos, gerando uma versão brasileira do VIA-IS similar à original. As análises de estrutura fatorial e descritivas foram realizadas com uma amostra de 1.975 brasileiros, com média de idade de 35,0 anos (DP $=10,8)$. Os resultados encontrados sugerem o uso do instrumento como uma medida unidimensional de potencialidades. A amostra apresentou médias altas de escores em todas as forças avaliadas, com mulheres relatando escores mais altos que homens, na maioria delas. São discutidas implicações para a avaliação em Psicologia Positiva de forças e virtudes com o VIA-IS em contexto brasileiro. Palavras-chave: inventário de forças, forças de caráter, virtudes, psicologia positiva, estrutura fatorial
\end{abstract}

\section{Brazilian Adaptation and Factor Structure of the 240-item VIA Inventory of Strengths Scale}

\begin{abstract}
The aims of this study were: (1) to perform the cross-cultural adaptation of the 240-item VIA Inventory of Strengths (VIA-IS) to use in Brazil; (2) to investigate the factor structure of the VIA-IS in a Brazilian sample; and (3) to conduct descriptive analyses of strengths scores in the VIA-IS in the Brazilian sample. The cross-cultural adaptation provided a Brazilian-Portuguese version of the VIA-IS that is similar to the original version of the instrument. The descriptive and factor structure analysis were conducted in a sample of 1.975 Brazilians, with a mean age of 35.0 years old $(S D=10.8)$. Results suggest that the instrument should be used as a unidimensional measure of strengths and virtues. The sample presented high scores in all strengths assessed, and women scored higher than men in the majority of the strengths. We discuss implications for the assessment of strengths and virtues in Positive Psychology using the VIA-IS in the Brazilian context.

Keywords: inventory of strengths, strength of character, virtues, positive psychology, factor structure
\end{abstract}

Adaptación Brasileña y Estructura Factorial de la Escala 240-item VIA Inventory of Strengths

\begin{abstract}
Resumen
Este estudio tuvo como objetivo: (1) realizar la adaptación transcultural del inventario 240-item VIA Inventory of Strengths (VIA-IS) para su uso en Brasil; (2) investigar la estructura factorial del VIA-IS en una muestra brasileña; y (3) informar datos descriptivos de las puntuaciones de fuerzas en el VIA-IS en esa muestra. La adaptación de la escala para su uso en Brasil siguió pautas estandarizadas para la adaptación transcultural de instrumentos psicológicos, generando una versión brasileña del VIA-IS similar a la original. Los análisis de estructura factorial y descriptivas se llevaron a cabo en una muestra de 1.975 brasileños, con edad media de 35,0 años (SD = 10,8). Los resultados sugieren que el instrumento debe ser utilizado como una medida unidimensional de potencialidades. La muestra presentó medias altas de puntuaciones en todas las fuerzas evaluadas, y las mujeres tuvieron mayor puntuación que los hombres en la mayoría de ellas. Se discuten las implicaciones para la evaluación en Psicología Positiva de fuerzas y virtudes utilizando el VIA-IS en el contexto brasileño.

Palabras clave: inventario de fuerza, fuerza de carácter, virtudes, psicología positiva, estructura factorial
\end{abstract}

\section{Introdução}

O conceito de forças de caráter (character strengths) é um dos pilares do campo da Psicologia Positiva, na medida em que sugere a identificação e o desenvolvimento de potencialidades humanas. Como proposto pelos fundadores da área, essas características psicológicas positivas exercem papel fundamental na vivência de outros aspectos positivos (Seligman \& Csikszentmihalyi, 2000). De acordo com essa perspectiva, o indivíduo que conhece suas forças e virtudes pode impulsionar seu "florescimento", ou seja, é uma condição que pode permitir seu desenvolvimento pleno e saudável em todos os níveis - psicológico, biológico e social (Keyes \& Haidt, 2003). Investigações sobre forças pessoais vêm ganhando espaço e fundamentando intervenções nos campos terapêutico (Seligman, Rashid, \& Parks, 2006), educacional (Park \& Peterson, 2009) e organizacional (Peterson \& Park, 2006).

A partir de pesquisas iniciais, Peterson e Seligman (2004) identificaram seis valores presentes em escritos históricos de Filosofia e religião em diferentes culturas. 
Desses valores, posteriormente denominados virtudes - sabedoria e conhecimento, coragem, humanidade, justiça, temperança e transcendência - emergiram 24 principais forças de caráter, que estariam presentes em todo indivíduo, em maior ou menor grau. De acordo com os propositores, as virtudes seriam características centrais, universais, valorizadas por filósofos e religiosos, que emergiram de documentos históricos analisados (Dahlsgaard, Peterson, \& Seligman, 2005). Já as forças de caráter seriam os ingredientes psicológicos que definiriam as virtudes (Peterson \& Park, 2004).

As forças de caráter podem ser conceituadas como características positivas que se refletem em pensamentos, sentimentos e comportamentos. Apresentam-se em níveis distintos para cada indivíduo e podem ser mensuradas (Park, Peterson, \& Seligman, 2004). Além disso, as forças exerceriam papel fundamental na vivência de outros aspectos positivos, como bem-estar (Seligman \& Csikszentmihalyi, 2000). Para Peterson e Seligman (2004), as forças devem preencher alguns critérios para serem consideradas como tais. Esses critérios foram levantados a partir dos estudos teóricos iniciais (ver Peterson \& Seligman, 2004).

Entende-se, atualmente, que as forças são influenciadas tanto pelo contexto - família, escola, pares e comunidade - quanto pela hereditariedade (Park \& Peterson, 2009; Steger, Hicks, Kashdan, Krueger, \& Bouchard, 2007). Além disso, as forças têm-se mostrado relacionadas com os estágios do ciclo vital (Park \& Peterson, 2006a, 2006b; Park, Peterson, \& Seligman, 2006). Estudos com jovens e adultos sugerem que as forças de caráter são relativamente estáveis ao longo do tempo, o que é considerado por alguns autores como um indício de que poderiam ser traços (Park \& Peterson, 2009). Entretanto, mesmo que apresentem relativa estabilidade ao longo do tempo, não se pode afirmar que são imutáveis (Peterson, 2006).

O estudo das forças de caráter deu origem a um manual de forças, uma nova classificação que tem por ponto de partida as potencialidades humanas (Peterson \& Seligman, 2004). Da mesma forma que há classificações para terminologias patológicas, a classificação de forças - VIA Classification of Strengths (Tabela 1) - propõe-se a ser um contraponto a essa visão, evidenciando a existência de aspectos saudáveis no ser humano e ampliando o olhar exclusivamente direcionado anteriormente para psicopatologias (Snyder \& Lopez, 2009). No entanto, essa classificação é descritiva, e não prescritiva, não podendo ser considerada uma taxonomia de forças (Niemiec, 2013). Dessa classificação, por sua vez, foi estruturada uma medida de forças de caráter, a 240-item VIA Inventory of Strengths (VIA-IS). O instrumento é composto por 240 itens - dez itens por força de caráter mensurada - avaliados em uma escala de concordância de cinco pontos (indo de "não tem nada a ver comigo" a "tem tudo a ver comigo"). O preenchimento da escala gera um ranking das forças mais bem pontuadas, chamado pelos propositores de Assinatura de forças (Peterson \& Seligman, 2004). Pesquisadores salientam que esse ranking é dinâmico (Peterson \& Seligman, 2004) e pode ser desenvolvido (Park, 2009; Peterson \& Park, 2009).

Aplicações do instrumento 240-item VIA Inventory of Strengths em 54 diferentes países indicaram consistência interna adequada $(\alpha>0,70)$. Análises fatoriais e análises de componentes principais realizadas em estudos anteriores demonstraram diferentes agrupamentos de forças para a interpretação da estrutura do instrumento (Tabela 2). Alguns estudos sugerem uma solução de cinco agrupamentos (MacDonald, Bore, \& Munro, 2008; Ruch et al., 2010; Singh \& Choubisa, 2010), corroborando as primeiras análises realizadas (Peterson \& Seligman, 2004). Cabe salientar que Peterson e Seligman (2004), apesar de encontrarem soluções estatísticas de cinco fatores, optaram por propor um modelo de seis fatores - virtudes - baseado em achados teóricos. Em contrapartida, também foram encontradas soluções fatoriais unidimensionais (MacDonald et al., 2008) de três componentes (Shryack, Steger, Krueger, \& Kallie, 2010) e de quatro componentes (MacDonald et al., 2008; Brdar \& Kashdan, 2010; Shryack et al., 2010).

As forças e virtudes propõem-se a ser universais, ou seja, podem ser identificadas em diferentes culturas, países e sistemas de crença (Dahlsgaard et al.,2005; Park \& Peterson, 2010; Park et al., 2006). Embora as forças sejam atributos que podem ser observados em diferentes culturas, é esperado que seu ranking seja particular do contexto, considerando seus valores, atitudes e comportamentos específicos (Park, et al., 2006). Um estudo realizado com dados de 54 países, incluindo o Brasil, indicou que as forças mais bem pontuadas foram gentileza, justiça, honestidade, gratidão, pensamento crítico, capacidade de amar e ser amado, e bom humor. Em contrapartida, as forças menos pontuadas nessas nações foram temperança, prudência, modéstia e autorregulação (Park et al., 2006). Alguns países apresentaram rankings semelhantes, formando agrupamentos que podem ser justificados por similaridades culturais. Foi o caso do agrupamento de países nórdicos e a aproximação de resultados do Reino Unido, 
Tabela 1

Descrição das Forças e Agrupamento em Virtudes, Traduzida para Português Brasileiro (adaptado de Peterson \& Seligman, 2004, pp. 29-30)

\begin{tabular}{ll}
\hline Virtudes & Forças \\
\hline Sabedoria e & Criatividade, engenhosidade e originalidade (CEO): Pensar em novas formas de fazer as coisas. Não estar
\end{tabular}

Conhecimento satisfeito em fazer algo da forma convencional se é possível um modo melhor./Curiosidade e interesse no mundo (CIM): Ser curioso. Fazer questões e achar os assuntos fascinantes. Gostar de explorar e descobrir./Juízo, pensamento crítico e abertura a novas ideias (JPA): Pensar sobre as coisas e examiná-las de todos os lados. Não se precipitar em conclusões e se basear apenas em evidências sólidas para tomar decisões. Ser capaz de mudar de opinião./ Amor pela aprendizagem (APA): Gostar de aprender coisas novas, tanto em aula quanto por si próprio. Gostar de ir à escola, de ler, e de museus, em qualquer lugar que haja oportunidade de aprendizado./Sabedoria (Sab): Apesar de não se considerar sábio, ser considerado por amigos. Ser levado em conta por sua perspectiva, e para aconselhamentos. Ter visão de mundo que faz sentido para os outros e para si mesmo.

Coragem Coragem e bravura (CoB): Ser corajoso, não recuar diante de uma ameaça, desafio, dificuldade ou dor. Defender o que é certo mesmo que haja oposição. Agir de acordo com suas convicções./ Honestidade, autenticidade e sinceridade (HAS): Ser honesto, não apenas por falar a verdade, mas por viver de forma genuína e autêntica. Ter os pés no chão e não ser pretensioso; ser realista./Perseverança, assiduidade e diligência (PAD): Trabalhar duro para terminar o que começa. Terminar projetos no tempo previsto. Não se distrair quando trabalha e ter satisfação em completar uma tarefa./Deleite, Entusiasmo e Energia (DEE): Independentemente do que faça, fazer com empolgação e energia. Não fazer pela metade ou sem entusiasmo. Ver a vida como uma aventura.

Humanidade Capacidade de amar e ser amado (CAS): Valorizar relações próximas com outras pessoas, especialmente aquelas nas quais compartilhar e cuidar são recíprocos. Sentir-se próximo de quem se sente próximo a você./Bondade e generosidade (BoG): Ser bom e generoso para os outros, e nunca estar ocupado para apoiar alguém. Gostar de realizar boas ações para os outros, mesmo que não as conheça bem./ Inteligência social (InS): Estar consciente dos motivos e sentimentos das outras pessoas. Saber o que fazer para se adequar a diferentes situações sociais e para deixar as pessoas à vontade.

Justiça Cidadania, trabalho em equipe e lealdade (CTL): Sobressair-se como membro de um grupo. Ser leal e dedicado, fazer sua parte e trabalhar duro pelo sucesso do grupo./Liderança (Lid): Sobressair-se em tarefas de liderança: encorajar um grupo a realizar coisas e preservar a harmonia entre o grupo fazendo com que todos se sintam inclú́dos. Fazer um bom trabalho organizando atividades e realizando-as./ Integridade, igualdade e justiça (IIJ): Tratar todas as pessoas imparcialmente. Não permitir que sentimentos pessoais tendenciem decisões sobre os outros. Oferecer uma nova chance a qualquer um.

Temperança Perdão e misericórdia (PeM): Perdoar aqueles que erraram com você. Dar aos outros uma segunda chance. Prezar pela misericórdia, e não pela vingança./Modéstia e bumildade (MoH): Não procurar pelos holofotes, preferindo que realizações falem por elas mesmas. Não se considerar especial, ver que os outros reconhecem e valorizam sua modéstia./ Autorregulação e autocontrole (ArA): Regular conscientemente o que sente e o que faz. Ser disciplinado. Estar no controle de seus desejos e emoções, não vice-versa./Prudência, cuidado e discrição (PCD): Ser cuidadoso e fazer escolhas consistentemente prudentes. Não dizer ou fazer algo que possa se arrepender depois.

Transcendência Apreciação da beleza e excelência (ABE): Perceber e apreciar a beleza, a excelência e/ou o bom desempenho em todos os domínios da vida, da natureza à arte, da matemática à ciência, nas experiências cotidianas./Gratidão (Gra): Ser consciente das coisas boas que acontecem sem considerálas garantidas. Ser reconhecida como uma pessoa grata porque expressa seus agradecimentos./ Esperança, otimismo e visão de futuro (EOV): Esperar o melhor do futuro e trabalhar para alcançar. Acreditar que o futuro é algo que pode controlar./Bom humor e diversão (BHD): Gostar de rir e caçoar. Provocar sorrisos nas outras pessoas. Tentar ver o lado bom de todas as situações./ Espiritualidade, senso de propósito e fé (ESF): Ter crenças fortes e coerentes sobre um propósito maior e sobre o sentido do universo. Saber onde se encaixa nesta grande engrenagem. Ter crenças que modelam suas ações e são um recurso para confortá-lo. 
Tabela 2

Agrupamentos de Forças por Fatores, Propostos por Estudos que Realizaram Análises Fatoriais do Instrumento VIA-IS

\begin{tabular}{|c|c|c|}
\hline Publicação & Fatores encontrados & Forças ${ }^{a}$ \\
\hline \multirow[t]{5}{*}{ Peterson e Seligman (2004) } & Sabedoria e Conhecimento & CEO, CIM, JPA, APA, Sab \\
\hline & Coragem & CoB, HAS, PAD, DEE \\
\hline & Humanidade & CAA, BoG, InS \\
\hline & Justiça & CTL, Lid, IIJ \\
\hline & Temperança & PeM, MoH, ArA, PCD \\
\hline \multirow{5}{*}{$\begin{array}{l}\text { MacDonald, Bore, e Munro } \\
(2008)\end{array}$} & Forças de regulação & IIJ, MoH, PeM, PCD \\
\hline & Forças intelectuais & CEO, CIM, APA, Sab, ABE \\
\hline & Forças interpessoais & BoG, CAA, Lid, CTL, BHD \\
\hline & Forças emocionais & CoB, EOV, ArA, DEE \\
\hline & Forças teológicas & Gra, ESF \\
\hline \multirow[t]{4}{*}{ Brdar e Kashdan (2010) } & Forças interpessoais & IIJ, CTL, BoG, PeM, CAA, MoH, Lid, Gra \\
\hline & Forças de caráter & JPA, CEO, APA, InS, Valor \\
\hline & Vitalidade & DEE, ESF, CIM, BHD \\
\hline & Cautela & PCD, ArA, PAD, EOV, HAS \\
\hline \multirow{3}{*}{$\begin{array}{l}\text { Shryack, Steger, Krueger, e } \\
\text { Kallie (2010) }\end{array}$} & Forças intelectuais & CEO, CIM, JPA, IIJ, APA, InS, ABE, DEE, EOV \\
\hline & Forças interpessoais & BoG, CAA, Lid, IIJ, CTL, PeM, Gra, BHD \\
\hline & Forças de temperança & PAD, HAS, ArA, PCD, MoH \\
\hline \multirow[t]{5}{*}{ Singh e Choubisa (2010) } & Forças cívicas & IIJ, CTL, MoH, Lid, HAS, PCD \\
\hline & Forças de autoconfiança & PAD, ArA, EOV, ESF, DEE \\
\hline & Forças interpessoais & $\mathrm{BHD}, \mathrm{CoB}, \mathrm{BoG}$ \\
\hline & Forças intelectuais & CEO, JPA, APA, CIM, EOV \\
\hline & Forças teológicas & Gra, CAA, ABE, PeM \\
\hline Publicação & Fatores encontrados & Forças $^{a}$ \\
\hline \multirow[t]{5}{*}{ Ruch et al. (2010) } & Forças emocionais & CoB, DEE, CAA, InS, EOV, BHD \\
\hline & Forças interpessoais & CTL, IIJ, Lid, PeM, MoH \\
\hline & Forças de autocontrole & EOV, PAD, HAS, PCD, ArA \\
\hline & Forças intelectuais & CEO, CIM, JPA, APA \\
\hline & Forças teológicas & ABE, Gra, ESF \\
\hline
\end{tabular}

Nota. CEO: Criatividade, engenhosidade e originalidade; CIM: Curiosidade e interesse no mundo; JPA: Juízo, pensamento crítico e abertura a novas ideias; APA: Amor pela aprendizagem; Sab: Sabedoria; CoB: Coragem e bravura; HAS: Honestidade, autenticidade e sinceridade; PAD: Perseverança, assiduidade e diligência; DEE: Deleite, Entusiasmo e Energia; CAS: Capacidade de amar e ser amado; BoG: Bondade e generosidade; InS: Inteligência social; CTL: Cidadania, trabalho em equipe e lealdade; Lid: Liderança; IIJ: Integridade, igualdade e justiça; PeM: Perdão e misericórdia; MoH: Modéstia e humildade; ArA: Autorregulação e autocontrole; PCD: Prudência, cuidado e discrição; ABE: Apreciação da beleza e excelência; Gra: Gratidão; EOV: Esperança, otimismo e visão de futuro; BHD: Bom humor e diversão; ESF: Espiritualidade, senso de propósito e fé.

Austrália e Nova Zelândia (Park et al., 2006). Entretanto, os autores afirmam não ser possível generalizar resultados de cada nação para toda sua população, visto que cada indivíduo apresenta suas particularidades.

Esse dado é corroborado pelo estudo de Biswas-Diener (2006), que analisou forças de caráter de três diferentes culturas - Kenyan Maasai, Inughuit e estudantes universitários americanos. De acordo com os resultados, algumas forças, como Coragem e bravura, podem ser consideradas panculturais, ao passo que outras parecem mais relacionadas com aspectos particulares do contexto. A força Modéstia, por exemplo, 
apresentou baixos escores para americanos e Inughuits, o que pode estar relacionado mudanças culturais ao longo do tempo (Biswas-Diener, 2006; Ellsworth, 1994). Outro aspecto a ser considerado são episódios marcantes na história das nações, os quais podem afetar a forma como as pessoas avaliam a si mesmas e valorizam determinadas forças. É o caso do ataque terrorista de 11 de setembro nos Estados Unidos, associado a mudanças de pontuações de várias forças de caráter, entre elas Liderança, Esperança e Gentileza (Peterson \& Seligman, 2003).

Ainda com relação a aspectos associados às forças de caráter, estudos sugerem diferenças entre gênero (Peterson \& Seligman, 2004; Shimai, Otake, Park, Peterson, \& Seligman, 2006; Shryack et al., 2010). Enquanto as mulheres apresentariam maiores escores para forças como Amor pela aprendizagem, Capacidade de amar e ser amado, Gentileza, Apreciação da beleza, Trabalho em equipe e Gratidão, homens endossaram mais frequentemente as forças Coragem e bravura, Criatividade e Autorregulação.

O presente estudo teve como objetivos: (1) realizar o processo de adaptação transcultural do 240-item VIA Inventory of Strengths (VIA-IS) para uso no Brasil; (2) investigar a estrutura fatorial do VIA-IS em uma ampla amostra brasileira; e (3) reportar dados descritivos de escores de forças no VIA-IS para a amostra brasileira (total e por sexo). As análises deste estudo tiveram caráter exploratório, dado ser este o primeiro trabalho com o instrumento em contexto brasileiro.

\section{Método}

Processo de Tradução e Adaptação do Instrumento para Uso no Brasil

A escala 240-item VIA Inventory of Strengths (VIA-IS) foi traduzida e adaptada para utilização brasileira a partir de uma parceria com o Values in Action Institute on Character, estabelecida contratualmente em 2010, conforme sugerido por Gjersing et al. (2010). O preenchimento foi realizado de forma on-line pelo site www.viacharacter. org. Após recebimento dos dados, a tradução inglês-português foi conduzida por um pesquisador expert em Psicologia, nativo do Brasil e com fluência no idioma inglês. Essa tradução foi encaminhada a juiz, que realizou o processo de retrotradução. Após isso, um terceiro pesquisador expert, também com fluência em ambos os idiomas, realizou a comparação entre a versão original e o resultado dos processos de tradução e retrotradução. Esse método de adaptação de instrumento tem sido sugerido pela literatura, a fim de assegurar que a tradução esteja de acordo com aspectos culturais e contextuais (Borsa, Damásio, \& Bandeira, 2012). Identificou-se concordância gramatical em 222 itens, com pequenas variações de sinônimos (ex. "Pode-se confiar em minhas promessas" foi alterado para "Minhas promessas são confiáveis"). Foram sugeridas modificações de tradução em 18 itens que apresentaram discrepâncias (Tabela 3), a fim de respeitar particularidades do contexto brasileiro. As alterações foram definidas por consenso dos experts, finalizando o processo de tradução do instrumento.

\section{Participantes}

Participaram do estudo 1.975 indivíduos que responderam ao questionário em português-brasileiro no website da instituição parceira. A Tabela 4 apresenta a caracterização sociodemográfica da amostra. A coleta de dados foi realizada entre maio de 2010 e abril de 2013. Os interessados em preencher a escala 240-item VIA Inventory of Strengths (VIA-IS) adaptada para uso no Brasil acessaram o website do Institute on Character $\mathrm{e}$ automaticamente foram computados como participantes deste estudo.

\section{Instrumento}

A escala 240-item VIA Inventory of Strengths (VIA-IS) é uma medida de forças de caráter proposta por Peterson e Seligman (2004). O instrumento é composto por 240 itens que mensuram, em escala de concordância de cinco pontos ("não tem nada a ver comigo" a "tem tudo a ver comigo"), 24 diferentes forças de caráter (ver Tabela 1). A escala é preenchida unicamente em formato on-line. Sua versão original, em contexto americano, apresentou consistência interna adequada $(\alpha>$ 0,70) (Peterson \& Seligman, 2004).

\section{Procedimentos}

A versão traduzida e adaptada da escala foi disponibilizada no website da instituição parceira. Um convite de participação foi encaminhado, por e-mail, a uma rede de contatos previamente organizada. Além disso, interessados de todo o país puderam acessar o instrumento pelo portal da instituição. O link da escala também foi divulgado por profissionais parceiros e pela Associação de Psicologia Positiva da América Latina. Os dados coletados foram repassados pelo Institute on Character, para viabilizar as análises apresentadas neste estudo. A versão brasileira do instrumento continua disponível no mesmo website, na opção de idioma Portuguese Brazil. 
Tabela 3

Processo de Tradução da Escala "240-item VLA Inventory of Strengths" (VIA-IS) - Itens Discrepantes

\begin{tabular}{ll}
\hline Item original & Primeira tradução \\
\hline $\begin{array}{l}\text { I am very aware of my } \\
\text { surroundings. }\end{array}$ & $\begin{array}{l}\text { Eu percebo muito bem tudo o que es } \\
\text { à minha volta. }\end{array}$ \\
$\begin{array}{l}\text { I always have a broad outlook on } \\
\text { what is going on. }\end{array}$ & $\begin{array}{l}\text { Eu sempre tenho uma visão abrangent } \\
\text { da situação. }\end{array}$ \\
$\begin{array}{ll}\text { I always look on the bright side. } \\
\begin{array}{l}\text { I want to fully participate in life, not } \\
\text { just view it from the sidelines. }\end{array}\end{array}$ & $\begin{array}{l}\text { Eu quero participar inteiramente da } \\
\text { vida, não apenas ficar assistindo da } \\
\text { margem. }\end{array}$
\end{tabular}

I always let bygones be bygones. Acho que o que passou, passou.

I never approach things balfheartedly.

I work at my very best when I am a group member.

I always know what makes someone tick.

People describe me as "wise beyond my years."

I call for action while others talk.

I am always able to look at things and see the big picture.

I go to extremes to acknowledge people who are good to me.

I read all of the time.

Thinking things through is part of who I am.

I rarely try to get even.

I stick with whatever I decide to do.

I am in awe of simple things in life that others might take for granted

I believe that everyone should have a say.

I have been richly blessed in my life.
Sempre coloco meu coração nas coisas que faço.

Eu trabalho melhor em grupo.

Eu sempre sei o que faz alguém vibrar.

As pessoas me descrevem como muito sábio para e minha idade.

Eu chamo à ação enquanto outros discutem.

Eu sempre consigo olhar as coisas e ver o panorama geral.

Eu me excedo para mostrar reconhecimento às pessoas que são boas para mim.

Eu leio o tempo todo.

Eu sou uma pessoa ponderada.

Eu raramente tento "ficar quite" ou "acertar as contas" com os outros.

Eu mantenho o que quer que tenha decidido fazer.

Eu reverencio pequenas coisas na vida talvez sem importância para outros.

Acredito que todas as partes devam ter voz em uma questão.

Eu ouvi um "chamado" em minha vida.
Versão final - decisão dos experts

Eu sou muito consciente do ambiente que me cerca.

Eu sempre procuro olhar com uma perspectiva mais ampla para o que está acontecendo.

Eu sempre considero o lado bom das coisas.

Eu quero aproveitar a vida plenamente, não apenas observá-la de fora.

Eu sempre deixo o passado como passado.

Eu nunca invisto em algo sem entusiasmo.

Eu faço o meu melhor quando sou membro de um grupo.

Eu sempre sei o que faz alguém se incomodar.

As pessoas me descrevem como alguém a frente do meu tempo.

Eu trato de agir enquanto os outros conversam.

Eu sempre estou disposto a olhar para as coisas e vê-las mais amplamente.

Eu vou a extremos para mostrar reconhecimento a quem é bom para mim.

Eu tenho consciência de tudo o que se passou e do que ainda está por vir.

Pensar através dos fatos é parte de quem eu sou.

Eu raramente tento me vingar.

Eu me empenho em qualquer coisa que eu decida fazer.

Eu me surpreendo com coisas simples da vida que outras pessoas podem não perceber.

Acredito que todo mundo tem algo a dizer.

Eu tenho uma vocação na vida. 
Tabela 4

Caracterização Sociodemográfica da Amostra

\begin{tabular}{lc}
\hline Característica sociodemográfica & \\
\hline Sexo declarado $n(\%)$ & $1.935(98 \%)$ \\
Homens $n(\%)$ & $685(34,7 \%)$ \\
Mulheres $n(\%)$ & $1.250(63,3 \%)$ \\
Não declararam sexo $n(\%)$ & $40(2 \%)$ \\
\hline Idade declarada $n(\%)$ & $1.815(91,9 \%)$ \\
Faixa de idade na amostra (anos) & $18-82$ \\
Média $(D P)($ anos) & $35,0(D P=10,8)$ \\
Não declararam idade $n(\%)$ & $160(8,1 \%)$ \\
\hline País de residência declarado $n(\%)$ & $1.762(89,2 \%)$ \\
Brasil $n(\%)$ & $1.720(87 \%)$ \\
Outros países $n(\%)$ & $42(2 \%)$ \\
Não declararam país de residência $n(\%)$ & $213(10,8 \%)$ \\
\hline
\end{tabular}

\section{Resultados}

Os dados dos participantes brasileiros foram sumarizados a partir das médias dos itens que compõem cada um dos 24 escores de forças do VIA-IS. Salienta-se que todas as forças tiveram médias acima de três pontos. Esses escores de forças foram, em seguida, submetidos a análises fatoriais exploratórias (AFE) para investigação da estrutura fatorial de virtudes da versão brasileira do questionário. Foram utilizadas AFE ao invés de Análises Fatoriais Confirmatórias (AFC) por conta dos resultados inconsistentes quanto à estrutura fatorial do VIA-IS encontrados em estudos anteriores com o instrumento em outros países e culturas. As análises foram realizadas no software FACTOR 9.2 (Lorenzo-Seva \& Ferrando, 2006). A investigação da adequação da amostra para a AFE foi feita pela análise do índice de Kaiser-Meyer-Olkin (KMO) e pelo teste de esfericidade de Bartlett. Foram utilizados dois métodos diferentes para avaliar o número de fatores ideal para retenção: método de Hull (Lorenzo-Seva, Timmerman, \& Kiers, 2011), e método de análises paralelas (Horns, 1965). O método de rotação empregado nos casos de soluções com mais de um fator foi o Varimax. Cabe comentar que diferentes análises fatoriais foram conduzidas com métodos de extração e rotação adequados para fatores comuns e em acordo com estudos anteriores sobre o instrumento. Por questões de convergência (i.e. falha em indicar uma solução após o número estipulado de interações), procedeu-se as análises de componentes principais com rotação Varimax, embora não sejam os métodos mais adequados. Cargas fatoriais estatisticamente significativas $(\phi<0,05)$ e com valores acima de 0,40 foram consideradas relevantes para interpretação das forças pertencentes a cada fator extraído. O coeficiente de confiabilidade dos fatores foi avaliado pelo método de Mislevy e Bock (1990). Embora a maioria dos estudos anteriores com o VIA-IS tenha utilizado o coeficiente de consistência interna alfa de Cronbach como evidência de confiabilidade do instrumento, a opção pelo uso de outro método se deu pelo reconhecimento das limitações do coeficiente alfa quando da análise de instrumentos com grande número de itens (isto é, valores altos de alfa são encontrados quando há um número muito elevado de itens, ainda que não necessariamente $\mathrm{o}$ instrumento apresente real consistência interna), limitações essas inerentes à própria fórmula utilizada para o cálculo do coeficiente alfa e reconhecidas por Cronbach (1951).

As análises preliminares demonstraram adequação da amostra para a realização das Análises Fatoriais Exploratórias $(K M O=0,95$; e índice de Bartlett $=$ $35784,3 ; g l=276 ; p<0,001)$. O método de Hull sugeriu a extração de um fator único, enquanto o método de análises paralelas sugeriu a extração de três ou quatro fatores. As soluções de três e quatro fatores correspondem à média e percentil $95 \%$ dos autovalores simulados. A Tabela 5 apresenta os resultados encontrados para as três soluções testadas - unifatorial, de três e de quatro fatores - quanto às seguintes 
propriedades: (1) cargas fatoriais de cada um dos escores das forças agrupadas pela divisão original do VIA-IS (Peterson \& Seligman, 2004); (2) índices de confiabilidade de cada fator; e (3) porcentagem de variância explicada por cada fator.

$\mathrm{Na}$ solução de três fatores, pode-se notar que o primeiro fator agrupou forças relacionadas à regulação e à criticidade, sugerindo aproximação com a terminologia Forças de regulação (MacDonald et al., 2008), Forças de autocontrole (Ruch et al., 2010) e Cautela (Brdar \& Kashdan, 2010). O segundo fator reuniu forças associadas a tipos de interesse ou envolvimento, o que pode se aproximar das ideias propostas nas virtudes Sabedoria e conhecimento (Peterson \& Seligman, 2004) e Forças intelectuais (MacDonald et al., 2008; Ruch et al., 2010; Shryack et al., 2010; Singh \& Choubisa, 2010). Já o terceiro fator agrupou forças relativas à socialização e à empatia, tendo aproximação com a virtude Forças interpessoais (Brdar \& Kashdan, 2010; MacDonald et al., 2008; Ruch et al., 2010; Shryack et al., 2010; Singh \& Choubisa, 2010). A força Perdão e Misericórdia não apresentou cargas fatoriais satisfatórias para nenhum fator.

A solução de quatro fatores indicou agrupamentos semelhantes. O quarto fator relacionou forças associadas à perspectiva e ao futuro, podendo se aproximar do conceito Temperança (Peterson \& Seligman, 2004; Shryack et al., 2010).

Além de Análises Fatoriais Exploratórias do instrumento VIA-IS, também foram realizadas análises descritivas de médias de pontuação das forças na amostra brasileira. Pode-se perceber que a força Integridade, igualdade e justiça apresentou maior média geral $(M=4,11, D P=0,50)$, enquanto a força Autorregulação e autocontrole teve a menor média $(M=$ 3,33, $D P=0,65)$ no estudo brasileiro. Salienta-se também que a força Espiritualidade, senso de propósito e fé apresentou maior desvio padrão entre os resultados gerais, o que indica maior dispersão dessa força na amostra brasileira.

As análises descritivas por sexo mostraram que as forças Criatividade, engenhosidade e originalidade, Curiosidade e interesse no mundo, Bom humor e diversão e Juízo, pensamento crítico e abertura a novas experiências foram mais bem pontuadas pelos homens. As mulheres demostraram pontuação mais alta nas demais forças. Salienta-se novamente o alto valor de desvio padrão, para ambos os sexos na forçaEspiritualidade, senso de propósito e fé. Os dados estão detalhados na tabela a seguir (Tabela 6).

\section{Discussão}

Os resultados da amostra brasileira do VIA-IS indicaram três possíveis interpretações para a estrutura fatorial do instrumento, a depender do tipo de método utilizado para indicação do melhor número de fatores a ser retido: 1) uma solução unifatorial (a partir do método de Hull); 2) uma solução de três fatores; ou 3) uma solução de quatro fatores (itens 2 e 3 extraídos a partir do método de análises paralelas). A solução unifatorial está em consonância com os resultados de estudos anteriores com o VIA-IS (e.g., Brdar \& Kashdan, 2009; MacDonald et al., 2008). Além disso, outro estudo realizado na África do Sul com a versão para jovens do instrumento (VIA-Youth) também apresentou solução unifatorial (Van Eeden, Wissing, Dreyer, Park, \& Peterson, 2008).

Já as soluções multifatoriais encontradas no presente estudo apresentaram uma distribuição de forças entre os fatores, relativamente distinta à estrutura de seis fatores original (Peterson \& Seligman, 2004). Esse resultado é similar aos resultados apresentados por outros estudos (Brdar \& Kashdan, 2010; MacDonald et al., 2008; Ruch et al., 2010; Shryack et al., 2010; Singh \& Choubisa, 2010), que também não replicaram a estrutura proposta no modelo original do VIA-IS.

Mesmo as forças extraídas por fator, nos diferentes estudos, não coincidem entre si, ainda que existam soluções com mesmo número de fatores. A Tabela 2 apresenta a comparação das soluções multifatoriais verificadas previamente na literatura, evidenciando a inconsistência entre as soluções multifatoriais encontradas em diversos estudos (Brdar \& Kashdan, 2010; MacDonald et al., 2008; Ruch et al., 2010; Shryack et al., 2010; Singh \& Choubisa, 2010). Os resultados do presente estudo também corroboram essas evidências. Isso sugere diferentes interpretações sobre cada fator extraído a depender da cultura em que o instrumento VIA-IS foi aplicado. De fato, mesmo o agrupamento em virtudes, proposto teoricamente no modelo original, não foi confirmado empiricamente pelos autores em análises fatoriais (Peterson \& Seligman, 2004), sugerindo a necessidade de aprofundar estudos psicométricos do instrumento.

As soluções multifatoriais, neste estudo, apresentaram índices de confiabilidade adequados. No entanto, é preciso levar em conta a presença de diversas forças com cargas cruzadas (i.e., cargas maiores do que 0,40 em mais de um fator) tanto na solução de três fatores (10 forças com cargas cruzadas) quanto 
Tabela 5

Extração de Fatores com o Método de Análises Paralelas e o Método de Hull

\begin{tabular}{|c|c|c|c|c|c|c|c|c|}
\hline \multirow{2}{*}{ Forças $^{a}$} & \multirow{2}{*}{1 Fator } & \multicolumn{3}{|c|}{3 Fatores } & \multicolumn{4}{|c|}{4 Fatores } \\
\hline & & F1 & F2 & F3 & F1 & $\mathrm{F} 2$ & F3 & F4 \\
\hline CEO & 0,64 & 0,18 & 0,81 & 0,10 & 0,82 & 0,19 & 0,16 & 0,03 \\
\hline CIM & 0,77 & 0,22 & 0,79 & 0,30 & 0,75 & 0,19 & 0,24 & 0,34 \\
\hline JPA & 0,70 & 0,68 & 0,45 & 0,15 & 0,46 & 0,70 & 0,22 & $-0,01$ \\
\hline APA & 0,68 & 0,27 & 0,70 & 0,20 & 0,67 & 0,25 & 0,16 & 0,28 \\
\hline Sab & 0,75 & 0,43 & 0,66 & 0,22 & 0,65 & 0,44 & 0,26 & 0,11 \\
\hline $\mathrm{CoB}$ & 0,72 & 0,43 & 0,66 & 0,18 & 0,63 & 0,40 & 0,15 & 0,27 \\
\hline PAD & 0,68 & 0,71 & 0,36 & 0,18 & 0,31 & 0,67 & 0,09 & 0,36 \\
\hline HAS & 0,69 & 0,75 & 0,19 & 0,35 & 0,17 & 0,75 & 0,34 & 0,18 \\
\hline DEE & 0,78 & 0,22 & 0,73 & 0,37 & 0,68 & 0,17 & 0,28 & 0,42 \\
\hline CAS & 0,67 & 0,11 & 0,37 & 0,68 & 0,30 & 0,07 & 0,57 & 0,45 \\
\hline BoG & 0,69 & 0,11 & 0,31 & 0,77 & 0,28 & 0,11 & 0,77 & 0,21 \\
\hline InS & 0,76 & 0,23 & 0,61 & 0,47 & 0,59 & 0,23 & 0,49 & 0,17 \\
\hline CTL & 0,77 & 0,37 & 0,27 & 0,72 & 0,24 & 0,36 & 0,72 & 0,22 \\
\hline IIJ & 0,77 & 0,43 & 0,27 & 0,68 & 0,24 & 0,43 & 0,70 & 0,17 \\
\hline Lid & 0,78 & 0,33 & 0,41 & 0,62 & 0,40 & 0,34 & 0,67 & 0,11 \\
\hline PeM & 0,61 & 0,20 & 0,22 & 0,20 & 0,17 & 0,18 & 0,58 & 0,34 \\
\hline $\mathrm{MoH}$ & 0,49 & 0,43 & $-0,14$ & 0,64 & $-0,19$ & 0,41 & 0,57 & 0,30 \\
\hline PCD & 0,58 & 0,81 & 0,09 & 0,23 & 0,08 & 0,80 & 0,22 & 0,14 \\
\hline ArA & 0,60 & 0,74 & 0,26 & 0,14 & 0,23 & 0,71 & 0,08 & 0,27 \\
\hline $\mathrm{ABE}$ & 0,58 & 0,06 & 0,51 & 0,42 & 0,47 & 0,04 & 0,38 & 0,27 \\
\hline Gra & 0,77 & 0,32 & 0,40 & 0,62 & 0,30 & 0,24 & 0,42 & 0,68 \\
\hline EOV & 0,78 & 0,41 & 0,57 & 0,37 & 0,49 & 0,34 & 0,21 & 0,57 \\
\hline BHD & 0,53 & $-0,17$ & 0,55 & 0,49 & 0,54 & $-0,15$ & 0,53 & 0,07 \\
\hline ESF & 0,60 & 0,33 & 0,28 & 0,48 & 0,16 & 0,22 & 0,21 & 0,79 \\
\hline Coeficiente de confiabilidade & 0,96 & 0,94 & 0,94 & 0,92 & 0,93 & 0,92 & 0,93 & 0,92 \\
\hline Variância explicada & 0,49 & 0,49 & 0,08 & 0,07 & 0,49 & 0,08 & 0,07 & 0,04 \\
\hline
\end{tabular}

Nota. CEO: Criatividade, engenhosidade e originalidade; CIM: Curiosidade e interesse no mundo; JPA: Juízo, pensamento crítico e abertura a novas ideias; APA: Amor pela aprendizagem; Sab: Sabedoria; CoB: Coragem e bravura; HAS: Honestidade, autenticidade e sinceridade; PAD: Perseverança, assiduidade e diligência; DEE: Deleite, Entusiasmo e Energia; CAS: Capacidade de amar e ser amado; BoG: Bondade e generosidade; InS: Inteligência social; CTL: Cidadania, trabalho em equipe e lealdade; Lid: Liderança; IIJ: Integridade, igualdade e justiça; PeM: Perdão e misericórdia; MoH: Modéstia e humildade; ArA: Autorregulação e autocontrole; PCD: Prudência, cuidado e discrição; ABE: Apreciação da beleza e excelência; Gra: Gratidão; EOV: Esperança, otimismo e visão de futuro; BHD: Bom humor e diversão; ESF: Espiritualidade, senso de propósito e fé.

na de quatro fatores (nove forças com cargas cruzadas). Além disso, o percentual de variância explicada pelos outros fatores que não o primeiro foi bastante baixo $(<1,0 \%$ para todos os outros fatores). Em conjunto, tais resultados parecem indicar a necessidade de reestruturar a divisão multifatorial do instrumento em virtudes.
Tendo em vista os frágeis resultados extraídos a partir das análises paralelas - que encontraram soluções multifatoriais - e a robustez do método de Hull, a solução unifatorial foi apontada, neste estudo, como melhor resultado. Adotar uma solução unifatorial no uso do VIA-IS implica um entendimento de que todas as forças para os indivíduos estão interligadas de tal forma 
Tabela 6

Escores Descritivos das Forças numa Amostra Brasileira - Valores de Média e Desvio Padrão Gerais e por Sexo

\begin{tabular}{cccccccc}
\hline Forças $^{a}$ & $\begin{array}{c}\text { Total } \\
\text { M (DP) }\end{array}$ & $\begin{array}{c}\text { Homens M } \\
(\mathrm{DP})\end{array}$ & $\begin{array}{c}\text { Mulheres M } \\
(\mathrm{DP})\end{array}$ & Forças $^{\mathrm{a}}$ & $\begin{array}{c}\text { Total } \\
\text { M (DP) }\end{array}$ & $\begin{array}{c}\text { Homens M } \\
(\mathrm{DP})\end{array}$ & $\begin{array}{c}\text { Mulheres M } \\
(\mathrm{DP})\end{array}$ \\
\hline ABE & $3,80(0,65)$ & $3,72(0,65)$ & $3,83(0,64)$ & BHD & $3,63(0,75)$ & $3,69(0,75)$ & $3,59(0,74)$ \\
CoB & $3,69(0,57)$ & $3,67(0,59)$ & $3,70(0,56)$ & PAD & $3,71(0,70)$ & $3,65(0,73)$ & $3,74(0,68)$ \\
CAS & $3,92(0,56)$ & $3,83(0,57)$ & $3,96(0,55)$ & JPA & $3,99(0,51)$ & $4,00(0,51)$ & $3,97(0,51)$ \\
PCD & $3,76(0,57)$ & $3,69(0,58)$ & $3,80(0,57)$ & BoG & $4,05(0,53)$ & $3,92(0,58)$ & $4,10(0,49)$ \\
CTL & $3,90(0,52)$ & $3,85(0,56)$ & $3,92(0,50)$ & Lid & $3,86(0,58)$ & $3,84(0,59)$ & $3,87(0,57)$ \\
CEO & $3,79(0,69)$ & $3,94(0,64)$ & $3,71(0,71)$ & APA & $3,75(0,63)$ & $3,67(0,66)$ & $3,78(0,61)$ \\
CIM & $3,89(0,54)$ & $3,89(0,55)$ & $3,88(0,54)$ & MoH & $3,47(0,64)$ & $3,40(0,68)$ & $3,50(0,61)$ \\
IIJ & $\mathbf{4 , 1 1 ( 0 , 5 0 )}$ & $4,08(0,52)$ & $4,13(0,48)$ & Sab & $3,76(0,54)$ & $3,76(0,55)$ & $3,75(0,53)$ \\
PeM & $3,66(0,67)$ & $3,64(0,69)$ & $3,67(0,66)$ & ArA & $\mathbf{3 , 3 3 ( 0 , 6 5 )}$ & $3,36(0,64)$ & $3,31(0,65)$ \\
Gra & $3,94(0,63)$ & $3,81(0,67)$ & $4,00(0,60)$ & InS & $3,79(0,53)$ & $3,72(0,57)$ & $3,81(0,49)$ \\
HAS & $3,97(0,50)$ & $3,88(0,54)$ & $4,01(0,47)$ & ESF & $3,50(0,97)$ & $3,38(1,02)$ & $3,55(0,94)$ \\
EOV & $3,85(0,67)$ & $3,80(0,68)$ & $3,86(0,66)$ & DEE & $3,81(0,64)$ & $3,78(0,67)$ & $3,82(0,64)$ \\
\hline
\end{tabular}

Nota. CEO: Criatividade, engenhosidade e originalidade; CIM: Curiosidade e interesse no mundo; JPA: Juízo, pensamento crítico e abertura a novas ideias; APA: Amor pela aprendizagem; Sab: Sabedoria; CoB: Coragem e bravura; HAS: Honestidade, autenticidade e sinceridade; PAD: Perseverança, assiduidade e diligência; DEE: Deleite, Entusiasmo e Energia; CAS: Capacidade de amar e ser amado; BoG: Bondade e generosidade; InS: Inteligência social; CTL: Cidadania, trabalho em equipe e lealdade; Lid: Liderança; IIJ: Integridade, igualdade e justiça; PeM: Perdão e misericórdia; MoH: Modéstia e humildade; ArA: Autorregulação e autocontrole; PCD: Prudência, cuidado e discrição; ABE: Apreciação da beleza e excelência; Gra: Gratidão; EOV: Esperança, otimismo e visão de futuro; BHD: Bom humor e diversão; ESF: Espiritualidade, senso de propósito e fé.

que não seria empiricamente apropriado separá-las em virtudes. Nesse sentido, o instrumento VIA-IS ofereceria um escore global de forças. O fato de as forças de caráter terem surgido de análises teóricas, e não propriamente da extração de fatores de uma escala, pode indicar que o agrupamento sugerido pelos propositores - formando o conceito de virtudes - é frágil e necessita de investigações futuras.

\section{Considerações Finais}

Este estudo apresentou o processo de tradução e evidências de validade para utilização do VIA-IS no Brasil. Cabe salientar, contudo, que alguns pontos não foram contemplados nas análises realizadas. Dados sociodemográficos, tais como escolaridade e renda, não foram incluídos pela ausência dessas informações no banco de dados repassado aos autores. Além disso, também não puderam ser verificadas diferenças regionais das forças no contexto brasileiro, o que poderia contribuir para uma compreensão bioecológica das forças de caráter.
A versão brasileira do VIA-IS pode ser um instrumento útil para auxiliar na avaliação de forças de caráter tanto no contexto de pesquisa quanto no contexto clínico. Embora os resultados trazidos pelo instrumento não devam ser interpretados como prescrições de atendimento ou para psicodiagnóstico, a escala é valida para complementar informações sobre potencialidades da pessoa. Em contexto clínico, nenhuma avaliação psicológica para investigar as potencialidades dos indivíduos deve se restringir ao uso de um único instrumento. Nesse contexto, é necessário complementar os dados oferecidos pelo VIA-IS com dados provenientes de outras fontes, como entrevistas clínicas ou outros instrumentos psicológicos validados para uso no Brasil.

Por fim, cabe salientar que as evidências empíricas apontam para o fato de que o VIA-IS oferece um escore válido e confiável de avaliação global de potencialidades dos indivíduos. Aqueles que pretendem utilizar o instrumento para avaliação de forças específicas ou agrupamentos delas devem ter cautela no processo de interpretação dos resultados, visto que soluções multifatoriais para esse instrumento são mais frágeis em termos psicométricos. 


\section{Referências}

Biswas-Diener, R. (2006). From the equator to the north pole: A study of character strengths. Journal of Hapiness Studies, 7, 293-310. doi: 10.1007/ s10902-005-3646-8

Borsa, J. C., Damásio, B. F., \& Bandeira, D. R. (2012). Adaptação e validação de instrumentos psicológicos entre culturas: Algumas considerações. Paidéia, 22(53), 423-432. doi: 10.1590/1982-43272253201314

Brdar, B., \& Kashdan, T. (2009). Character strengths and well-being in Croatia: An empirical investigation of structure and correlates. Journal of Research in Personality, 44(1), 151-154. doi: 10.1016/j.jrp.2009.12.001

Cronbach, L. J. (1951). Coefficient alpha and the internal structure of tests. Psychometrika, 16, 297-334. Recuperado de http://kttm.hoasen.edu.vn/sites/ default/files/2011/12/22/cronbach_1951_coefficient_alpha.pdf

Dahlsgaard, K., Peterson, C., \& Seligman, M. E. P. (2005). Shared virtue: The convergence of valued human strengths across culture and history. Review of General Psychology, 9(3), 203-213. doi: 10.1037/1089-2680.9.3.203

Ellsworth, P.C. (1994). Sense, culture, and sensibility. In S. Kitiyama \& H. R. Markus (Eds.), Emotion and Culture: Empirical Studies of Mutual Influence (pp. 23-50). Washington, DC: American Psychological Association.

Gjersing, L., Caplehorn, J. R. M., \& Clausen, T. (2010). Cross-cultural adaptation of research instruments: Language, setting, time and statistical considerations. BMC Medical Research Methodology, 10, 13. doi:10.1186/1471-2288-10-13

Horn, J. L. (1965). A rationale and test for the number of factors in factor analysis. Psychometrika, 30, 179185. doi: $10.1007 / \mathrm{BF} 02289447$

Keyes, C. L. M., \& Haidt, J. (2003). Flourishing: positive psychology and the life well lived. Washington, DC: American Psychological Association.

Lorenzo-Seva, U., \& Ferrando, P. J. (2006). FACTOR: A computer program to fit the exploratory factor analysis model. Behavior Research Methods, 38(1), 88-91. doi: 10.3758/BF03192753
Lorenzo-Seva, U., Timmerman, M. E., \& Kiers, H. A. (2011). The hull method for selecting the number of common factors. Multivariate Behavioral Research, 46(2), 340-364. doi: 10.1080/00273171.2011.564527

Macdonald, Bore, \& Munro. (2008). Values in action scale and the Big 5: An empirical indication of structure. Journal of Research in Personality, 42, 787799. doi: 10.1016/j.jrp.2007.10.003

Mislevy, R. J., \& Bock, R. D. (1990). BILOG: Item analysis and test scoring with binary logistic models. Chicago, IL: Scientific Software.

Niemiec, R. M. (2013). VIA Character Strengths: Research and Practice (The First 10 Years). In H. H. Knoop \& A. Delle-Fave (Eds.), Well-Being and Cultures (pp. 11-29). New York, NY: Springer.

Park, N. (2009). Building strengths of character: Keys to positive youth development. Reclaiming Children and Youth, 18, 42-47. Recuperado de http://www. fatih.edu.tr/ hugur/kindnes/BUILDING $\% 20$ STRENGTH $\% 20$ OF\%20CHARACTER.PDF

Park, N., \& Peterson, C. (2006a). Character strengths and happiness among young children:

Content analysis of parental descriptions. Journal of Happiness Studies, 7, 323-341. doi: 10.1007/ s10902-005-3648-6

Park, N., \& Peterson, C. (2006b). Moral competence and character strengths among adolescents: The development and validation of the Values in Action Inventory of Strengths for Youth. Journal of Adolescence, 29, 891-905. doi: 10.1016/j. adolescence.2006.04.011

Park, N., \& Peterson, C. (2009). Character Strengths: Research and practice. Journal of College and Character, 10(4), 1-10. doi: 10.2202/1940-1639.1042

Park, N., \& Peterson, C. (2010). Does it matter where we live? The urban psychology of character strengths. American Psychologist, 65(6), 535-547. doi: 10.1037/ a0019621

Park, N., Peterson, C., \& Seligman, M. E. P. (2004). Strengths of character and well-being, Journal of Social \& Clinical Psychology, 23, 603-619. doi: 10.1521/ jscp.23.5.603.50748

Park, N., Peterson, C., \& Seligman, M. E. P. (2006). Character strengths in fifty-four nations and the 
fifty US states. Journal of Positive Psychology, 1, 118129. doi:10.1080/17439760600619567

Peterson, C. (2006). A primer in positive psychology. New York: Oxford University Press.

Peterson, C., \& Park, N. (2004). Classification and measurement of character strengths: Implications for practice. In P. A. Linley; \& S. Joseph (Eds.), Positive psychology in practice (pp. 433-446). Hoboken, NJ: Wiley.

Peterson, C., \& Park, N. (2006). Character strengths in organizations. Journal of Organizational Behavior, 27, 1149-1154. doi: 10.1002/job.398

Peterson, G., \& Park, N. (2009). Classifying and measuring strengths of character. In S. J. Lopez \& G. R. Snyder (Eds.), Oxford handbook of positive psychology, (2nd ed., pp. 25-33). New York, NY: Oxford University Press.

Peterson, C., \& M.E.P., Seligman (2003). Character strengths before and after September 11. Psychological Science, 14, 381-384. doi: 10.1111/1467-9280.24482

Peterson, C., \& Seligman, M. E. P. (2004). Character strengths and virtues: a handbook and classification. Washington, DC: American Psychological Association.

Seligman, M. E. P., \& Csikszentmihalyi, M. (2000). Positive psychology: an introduction. American Psychologist, 55, 5-14. doi: 10.1037/0003-066X.55.1.5

Seligman, M., Rashid, T., \& Parks, A. C. (2006). Positive Psychotherapy. American Psychologist, 61, 774-788. doi: 10.1037/0003-066X.61.8.774

Seligman, M. E. P. (2002). Positive psychology, positive prevention, and positive therapy. In C. R. Snyder \& S. J. Lopez (Eds.), Handbook of positive psychology (pp. 3-9). New York, NY: Oxford University Press.

Shimai, S., Otake, K., Park, N., Peterson, C., \& Seligman, M. E. P. (2006). Convergence of character strengths in american and japanese young adults.
Journal of Hapiness Studies, 7(3), 311-322. doi: 10.1007/s10902-005-3647-7

Shryack, J., Steger, M. F., Krueger, R. F., \& Kallie, C. S. (2010). The structure of virtue: An empirical investigation of the dimensionality of the virtues in action inventory of strengths. Personality and Individual Differences, 48, 714-719. doi: 10.1016/j. paid.2010.01.007

Singh, K., \& Choubisa, R. (2010). Empirical validation of values in Action-Inventory of Strengths (VIA-IS) in Indian context. Psychological Studies, 55, 151-158. doi: 10.1007/s12646-010-0015-4

Snyder, C. R., \& Lopez, S. J. (2009). Psicologia Positiva: Uma abordagem cientifica e prática das qualidades bumanas (R. C. Costa, Trad.). São Paulo, SP: Artmed.

Steger, M. F., Hicks, B., Kashdan, T. B., Krueger, R. F., \& Bouchard, T. J., Jr. (2007). Genetic and environmental influences on the positive traits of the Values in Action classification, and biometric covariance with normal personality. Journal of Research in Personality, 41, 524-539. doi: 10.1016/j. jrp.2006.06.002

Ruch, W., Proyer, R. T., Harzer, C., Park, N., Peterson, C., \& Seligman, M. E. P. (2010). Values in Action Inventory of Strengths (VIA-IS): Adaptation and validation of the German Version and the development of a peer-rating form. Journal of Individual Differences, 31, 138-149. doi: 10.1027/1614-0001/ a000022.

Van Eeden, C., Wissing, M. P., Dreyer, J., Park, N., \& Peterson, C. (2008). Validation of the values in action inventory of strengths for youth among South African learners. Journal of Psychology in Africa, 18, 143-154. doi: 10.1080/14330237.2008.10820181

Recebido: 04/03/2014

Primeira reformulação: 24/11/2014

Aprovado: 11/02/2015 
Sobre os autores:

Bruna Larissa Seibel é psicóloga, mestre em Psicologia pela Universidade Federal do Rio Grande do Sul e doutoranda pela mesma instituição. É membro do Centro de Estudos Psicológicos CEP-Rua e pesquisa temas relacionados à Psicologia Positiva e à Abordagem Familiar Sistêmica.

E-mail:brunaseibel@gmail.com

Diogo DeSousa é psicólogo, mestre em Psicologia pela Universidade Federal do Rio Grande do Sul, doutorando em Psicologia pela UFRGS e membro do Centro de Estudos Psicológicos CEP-Rua e do Programa de Transtornos de Ansiedade do Hospital de Clínicas de Porto Alegre. Instituto de Psicologia - UFRGS.

E-mail:diogo.a.sousa@gmail.com

Silvia Helena Koller é professora do Programa de Pós-Graduação da Universidade Federal do Rio Grande do Sul, doutora em Psicologia, pesquisadora 1A do CNPq e coordenadora do Centro de Estudos Psicológicos.

E-mail: silvia.koller@gmail.com

Contato com os autores:

Bruna Larissa Seibel

E-mail: brunaseibel@gmail.com

Rua/UFRGS - Rua Ramiro Barcelos, 2600/104, Porto Alegre/RS.

CEP: 90035-003 
